\title{
Atenção à saúde mental na atenção primária de saúde: uma revisão sistemática
}

\author{
Mental health care in primary health care: a systematic review \\ La atención de salud mental en la atención primaria de salud: una revisión sistemática
}

Recebido: 27/02/2021 | Revisado: 09/03/2021 | Aceito: 15/03/2021 | Publicado: 21/03/2021

\author{
Rita Adriana Melo Sterling \\ ORCID: https://orcid.org/0000-0002-9642-6772 \\ Faculdade CESUSC, Brasil \\ E-mail: ritasterling@faculdadecesusc.edu.br \\ Laura Faustino Gonçalves \\ ORCID: https://orcid.org/0000-0002-0043-4349 \\ Universidade Federal de Santa Catarina, Brasil \\ E-mail: laurafaustinog@ outlook.com \\ Patrícia Haas \\ ORCID: https://orcid.org/0000-0001-9797-7755 \\ Universidade Federal de Santa Catarina, Brasil \\ E-mail: patricia.haas@ufsc.br
}

\begin{abstract}
Resumo
Introdução: A atenção primária à saúde se apresenta como a principal porta de entrada do sistema de saúde, com isso, espera-se que as demandas de saúde mental também possam ser acolhidas, encaminhadas, acompanhadas e muitas vezes tratadas pelos profissionais que atuam nesse serviço. Objetivo: Verificar evidências científicas baseadas em evidências referentes à atenção à saúde mental na atenção primária à saúde. Metodologia: A busca por artigos científicos foi conduzida por dois pesquisadores independentes nas bases de dados Medline (Pubmed), LILACS, SciELO, Bireme, Web of Science e Scopus, sem restrição de idioma, período e localização. Foram inclusos na pesquisa estudos que obtiveram pontuação $\geq$ a 6 pontos segundo o protocolo para pontuação qualitativa proposto por Pithon et al. (2015). Resultados: Os estudos admitidos apontam para pacientes que obtiveram cuidados de saúde mental apenas do médico da atenção primária, bem como, avaliação de feedback consideraram os aplicativos úteis. Vinte e quatro $(68,5 \%)$ dos pacientes relataram o uso de Breathe2Relax e $24(68,5 \%)$ relataram o uso de autoajuda para Gerenciamento de Ansiedade. Conclusão: Um maior foco no cuidado centrado no paciente, requer uma maior integração entre atenção primária e o setor de saúde mental para assim, haver uma redução da fragmentação do cuidado e das disparidades nos resultados da saúde. Os aplicativos relacionados à saúde mental são aplicáveis e relevantes para os pacientes em ambientes integrados de atenção primária em sistemas de saúde de rede de segurança. Palavras-chave: Assistência à saúde mental; Saúde mental; Serviços de saúde mental; Atenção primária à saúde.
\end{abstract}

\begin{abstract}
Introduction: Primary health care presents itself as the main gateway to the health system, therefore, it is expected that the demands of mental health can also be welcomed, forwarded, monitored and often treated by professionals who work in this service. Objective: To verify scientific evidence based on evidence regarding mental health care in primary health care. Methodology: The search for scientific articles was conducted by two independent researchers in the databases Medline (Pubmed), LILACS, SciELO, Bireme, Web of Science and Scopus, without restriction of language, period and location. Studies that scored $\geq 6$ points according to the qualitative scoring protocol proposed by Pithon et al. (2015). Results: The admitted studies point to patients who obtained mental health care only from the primary care physician, as well as feedback assessment found the applications useful. Twenty-four (68.5\%) of the patients reported using Breathe2Relax and 24 (68.5\%) reported using self-help for Anxiety Management. Conclusion: A greater focus on patient-centered care requires greater integration between primary care and the mental health sector so that there is a reduction in the fragmentation of care and disparities in health outcomes. Mental health-related applications are applicable and relevant to patients in integrated primary care environments in safety net health systems.
\end{abstract}

Keywords: Mental health assistance; Mental health; Mental health services; Primary health care.

\section{Resumen}

Introducción: La atención primaria de salud se presenta como la principal puerta de entrada al sistema de salud, por lo que se espera que las demandas de la salud mental también sean acogidas, remitidas, monitoreadas y muchas veces tratadas por los profesionales que laboran en este servicio. Objetivo: Verificar la evidencia científica basada en evidencias sobre la atención de la salud mental en la atención primaria de salud. Metodología: La búsqueda de artículos científicos fue realizada por dos investigadores independientes en las bases de datos Medline (Pubmed), LILACS, SciELO, Bireme, Web of Science y Scopus, sin restricción de idioma, período y ubicación. Los estudios que 
puntuaron $\geq 6$ puntos según el protocolo de puntuación cualitativa propuesto por Pithon et al. (2015). Resultados: Los estudios admitidos apuntan a pacientes que obtuvieron atención de salud mental solo del médico de atención primaria, y la evaluación de retroalimentación encontró útiles las aplicaciones. Veinticuatro (68,5\%) de los pacientes informaron que usaban Breathe2Relax y $24(68,5 \%)$ informaron que usaban la autoayuda para el manejo de la ansiedad. Conclusión: Un mayor enfoque en la atención centrada en el paciente requiere una mayor integración entre la atención primaria y el sector de la salud mental para que haya una reducción en la fragmentación de la atención y las disparidades en los resultados de salud. Las aplicaciones relacionadas con la salud mental son aplicables y relevantes para los pacientes en entornos integrados de atención primaria en los sistemas de salud de la red de seguridad.

Palabras clave: Asistencia de salud mental; Salud mental; Servicios de salud mental; Primeros auxilios.

\section{Introdução}

A atenção primária à saúde (APS) é conceituada como o nível do sistema de saúde responsável por oferecer à comunidade os cuidados necessários para os seus problemas de saúde mais prevalentes. A APS deve ser também, o primeiro contato na rede assistencial dentro do sistema de saúde, caracterizando-se, principalmente pela continuidade e integralidade da atenção, além da coordenação da assistência dentro do próprio sistema, da atenção centrada na família, da orientação e participação comunitária e da competência cultural dos profissionais (Wenceslau, Leandro \& Francisco, 2015).

As equipes da atenção básica $(\mathrm{AB})$ são um importante recurso de enfrentamento de agravos à saúde e é inviável ponderar sobre saúde integral sem incluir a Saúde Mental (SM). É preconizado que aqueles que necessitam de acompanhamento devem ter o cuidado disponível em seu território e a $\mathrm{AB}$ é um importante articulador dos serviços da rede de SM (Carvalho \& Nóbrega, 2017). Salienta-se, também, que a continuidade do cuidado é um elemento central de uma atenção primária efetiva e quando há um relacionamento contínuo entre profissional de saúde e paciente, a qualidade dos serviços de SM na atenção primária tende a ser mais efetivo. Os serviços de atenção primária são, geralmente, os mais acessíveis, disponíveis e aceitos pela população (Wenceslau, Leandro \& Francisco, 2015)

Entende-se que a SM não está dissociada da saúde geral e suas demandas revelam-se em todos os serviços da rede de APS, especialmente na $\mathrm{AB}$. É necessário que o cuidado em SM seja incorporado à prática em todos os níveis de atenção, com intervenções capazes de considerar a subjetividade, a singularidade e a forma como o indivíduo percebe o mundo (Carvalho \& Nóbrega, 2017). São destacadas como as principais razões para integração da SM à atenção primária, à elevada carga de doença dos transtornos mentais; conexão entre problemas de saúde física e mental; enorme gap terapêutico dos transtornos mentais. Além disso, a atenção primária, em especial, oferece para a SM ampliação do acesso ao paciente com esta demanda; promoção dos direitos humanos; disponibilidade e custo-efetividade, além de, proporcionar bons resultados clínicos (Wenceslau, Leandro \& Francisco, 2015)

Compreender que a APS é a principal porta de entrada do sistema de saúde e almejar que demandas de SM também sejam resolvidas pelos profissionais da APS, e se necessário, realizar encaminhamento para unidade especializada consiste em um grande desafio para a população. Para que isto aconteça de forma positiva, é necessário que o acolhimento seja feito com maior cautela, justamente por entender que pacientes com alguma demanda de SM possa manifestar distorções cognitivas, sendo crucial à atenção ao acolhimento para que seja possível realizar devolutiva mais adequada ao paciente (Filho \& Bezerra, 2018).

Dessa forma, o acolhimento como dispositivo do processo de trabalho apresenta potencial para viabilizar o cuidado integral ao usuário, em busca da humanização na assistência. Para tanto, é imprescindível que os profissionais entendam a real importância do acolhimento, descaracterizando a visão de recepção, triagem e realização de encaminhamento sem adequada avaliação (Filho \& Bezerra, 2018). 
A partir do exposto, a presente pesquisa apresenta como objetivo verificar evidências científicas baseadas em evidências referentes à atenção à saúde mental na atenção primária à saúde, visando responder a seguinte pergunta norteadora de pesquisa: Como é realizada a atenção à saúde mental na atenção primária à saúde?

\section{Metodologia}

\section{Protocolo e registro}

A presente revisão foi conduzida conforme as recomendações PRISMA (Preferred Reporting Items for Systematic reviews and Meta-Analyses) (Moher et al., 2015). As buscas por artigos científicos foram conduzidas por dois pesquisadores independentes nas bases de dados eletrônicas MEDLINE (Pubmed), LILACS, SciELO, SCOPUS, WEB OF SCIENCE e BIREME, sem restrição de idioma, período e localização. A pesquisa foi estruturada e organizada na forma PICOS, que representa um acrônimo para População alvo, a Intervenção, Comparação e “Outcomes” (desfechos). População de interesse ou problema de saúde $(\mathrm{P})$ corresponde a pacientes; intervenção (I): psicologia; comparação (C): atenção à saúde mental; outcome $(\mathrm{O})$ : atenção primária de saúde; $(\mathrm{S})$ : estudo transversal, estudo observacional, relatos de caso, estudos de casocontrole, ensaios clínicos controlados, estudos de coorte.

Tabela 1. Descrição dos componentes do PICOS.

\begin{tabular}{l|l}
\hline \multicolumn{1}{c|}{ Acrônimo } & \multicolumn{1}{|c}{ Definição } \\
\hline $\mathbf{P}$ & Pacientes \\
\hline $\mathbf{I}$ & Psicologia \\
\hline $\mathbf{C}$ & Atenção à saúde mental \\
\hline $\mathbf{O}$ & Atenção Primária de Saúde \\
\hline $\mathbf{S}$ & $\begin{array}{l}\text { estudo transversal, } \\
\text { observacional, relatos de caso, } \\
\text { estudos de caso-controle, ensaios } \\
\text { clínicos controlados, estudos de } \\
\text { coorte. }\end{array}$ \\
\hline
\end{tabular}

Fonte: Desenvolvido pelos autores.

\section{Estratégia De Pesquisa}

Os descritores foram selecionados a partir dos vocabulários controlados Descritores em Ciências da Saúde (DeCS) e Medical Subject Heading Terms (MeSH), haja vista a sua grande utilização pela comunidade científica para a indexação de artigos na base de dados PubMed. Utilizou-se como estratégia de busca a combinação de descritor e operador booleano: (primary care) and (single health service) and (psychology) and (mental health). A busca ocorreu de forma concentrada em janeiro de 2021. Para complementar e evitar viés de risco foi realizada uma busca por literatura cinza no Google Scholar.

\section{Critérios De Elegibilidade}

Foram inclusos estudos sem restrição de idioma, período e localização. A Tabela 2 representa os critérios de inclusão e exclusão desenvolvidos nesta pesquisa. O estudo obteve pontuação 12 no protocolo modificado de Pithon et al. (2015) para avaliação da qualidade dos mesmos. 
Tabela 2. Síntese dos critérios de inclusão/exclusão.

\begin{tabular}{|c|c|}
\hline \multicolumn{2}{|c|}{ Critérios de Inclusão } \\
\hline Delineamento & $\begin{array}{c}\text { Relatos de casos } \\
\text { Estudos de casos e controle } \\
\text { Ensaios clínicos controlados } \\
\text { Estudos de coorte } \\
\text { Estudos em triagem } \\
\text { Estudos observacionais }\end{array}$ \\
\hline Localização & Sem Restrição \\
\hline Idioma & Sem restrição \\
\hline \multicolumn{2}{|c|}{ Critérios de Exclusão } \\
\hline Delineamento & $\begin{array}{c}\text { Cartas ao editor } \\
\text { Diretrizes } \\
\text { Revisões de literatura } \\
\text { Revisões sistemáticas } \\
\text { Meta-análises }\end{array}$ \\
\hline Estudos & $\begin{array}{c}\text { Estudos pouco claros } \\
\text { Mal descritos ou inadequados }\end{array}$ \\
\hline Forma de publicação & Apenas resumo \\
\hline
\end{tabular}

Fonte: Desenvolvido pelos autores.

\section{Risco de viés}

A qualidade dos métodos utilizados no estudo incluído foi avaliada pelo revisor de forma independente (PH), de acordo com a recomendação PRISMA (Moher et al., 2015). A avaliação priorizou a descrição clara das informações. Neste ponto, a revisão foi realizada às cegas, mascarando os nomes dos autores e revistas, evitando qualquer viés potencial e conflito de interesses.

\section{Critérios de Exclusão}

Foram excluídos estudos publicados nos formatos de Cartas ao editor, diretrizes, revisões de literatura, revisões narrativas, revisões sistemáticas, meta análises e resumos. Estudos indisponíveis na íntegra, também foram excluídos (Tabela 2).

\section{Análise dos dados}

A extração dos dados para o processo de elegibilidade dos estudos foi realizada utilizando-se uma ficha própria para revisão sistemática elaborada por dois pesquisadores em Programa Excel ${ }^{\circledR}$, na qual os dados extraídos foram adicionados por um dos pesquisadores e, então, conferidos por outro pesquisador. Inicialmente foram selecionados de acordo com o título; em seguida, os resumos foram analisados e apenas os que fossem potencialmente elegíveis foram selecionados. Com base nos resumos, artigos foram selecionados para leitura integral, foram admitidos os que atendiam a todos os critérios prédeterminados. 


\section{Forma de seleção dos estudos}

Inicialmente o revisor de elegibilidade (R e LFG) foi calibrado para a realização da revisão sistemática por PH. Após a calibração e esclarecimentos de dúvidas, os títulos e resumos foram examinados pelos revisores de elegibilidade (PH), de forma independente, os quais não estavam cegos para o nome dos autores e das revistas. Aqueles que apresentaram um título dentro do âmbito, mas os resumos não estavam disponíveis, também foram obtidos e analisados na íntegra. Foram excluídos estudos fora do âmbito proposto, relatos de caso, cartas ao editor e/ou editorial, revisões de literatura, índices, resumos e revisão sistemática, metanálise. Posteriormente, os estudos elegíveis preliminarmente tiveram o texto completo obtido e avaliado.

\section{Dados Coletados}

Após a triagem, o texto do artigo selecionado foi revisado e extraído de forma padronizada por dois autores (R e LFG) sob a supervisão de PH, identificando-se ano de publicação, local da pesquisa, idioma de publicação, tipo de estudo, amostra, método, resultado e conclusão do estudo.

\section{Resultado clínico}

O resultado clínico de interesse consistiu em verificar evidências científicas baseadas em evidências referentes à atenção à saúde mental na atenção primária à saúde. Aqueles que não utilizaram a abordagem definida não fizeram parte da amostra da revisão sistemática.

\section{Resultados e Discussão}

Inicialmente foram selecionados 175 artigos, dimensionado para 167 após exclusão por repetição; em seguida, os títulos e resumos foram analisados e 165 trabalhos foram excluídos pois não pertenciam ao escopo da proposta da pesquisa. Sendo então admitidos para a análise final dois artigos no qual todos foram inclusos na presente pesquisa (Figura 1). Os desenhos do estudo selecionado foram do tipo estudo transversal. 
Figura 1. Fluxograma de busca e análise dos artigos.

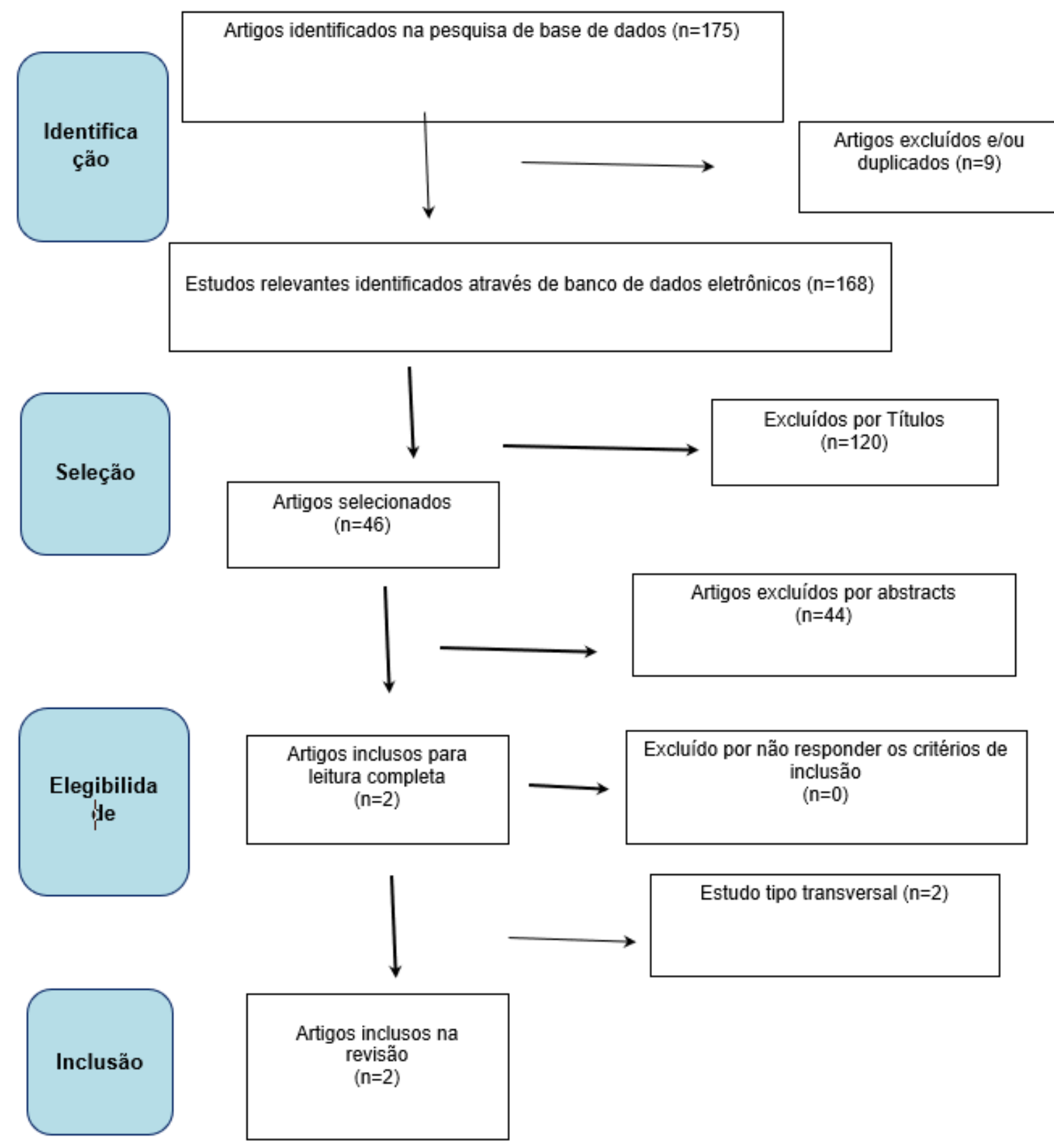

Fonte: Desenvolvido pelos autores.

A partir dos descritores eleitos, os bancos de dados foram consultados e foram obtidos os resultados disponibilizados na Tabela 3 . 
Tabela 3. Classificação das referências obtidas nas bases de dados Pubmed, Scielo, Lilacs, Web Of Science e Scopus.

\begin{tabular}{|c|c|c|c|c|c|}
\hline Descritores & $\begin{array}{l}\mathrm{N}^{\circ} \text { total } \\
\text { de } \\
\text { artigos }\end{array}$ & $\begin{array}{c}\mathrm{N}^{\circ} \text { de } \\
\text { Referências } \\
\text { excluídas }\end{array}$ & Motivo da exclusão & $\begin{array}{l}\mathrm{N}^{\circ} \text { de artigos } \\
\text { selecionados }\end{array}$ & $\begin{array}{c}\text { Banco de } \\
\text { dados }\end{array}$ \\
\hline $\begin{array}{l}\text { (primary care) and } \\
\text { (single health service) } \\
\text { and (psychology) and } \\
\text { (mental health) (primary } \\
\text { care) and (single health } \\
\text { service) and (psychology) } \\
\text { and (mental health) }\end{array}$ & 151 & 149 & $\begin{array}{l}\text { Excluídos por título } \\
\text { (100), excluídos por } \\
\text { resumo (40), } \\
\text { duplicados (9) }\end{array}$ & 2 & Pubmed \\
\hline $\begin{array}{l}\text { (primary care) and } \\
\text { (single health service) } \\
\text { and (psychology) and } \\
\text { (mental health) }\end{array}$ & 4 & 4 & $\begin{array}{l}\text { Excluídos por título } \\
\text { (4) }\end{array}$ & 0 & Lilacs \\
\hline $\begin{array}{l}\text { (primary care) and } \\
\text { (single health service) } \\
\text { and (psychology) and } \\
\text { (mental health) }\end{array}$ & 10 & 10 & $\begin{array}{l}\text { Excluídos por título } \\
(6), \text { excluídos por } \\
\text { resumo (4) }\end{array}$ & 0 & $\begin{array}{l}\text { Web of } \\
\text { Science }\end{array}$ \\
\hline $\begin{array}{l}\text { (primary care) and } \\
\text { (single health service) } \\
\text { and (psychology) and } \\
\text { (mental health) }\end{array}$ & 5 & 5 & $\begin{array}{l}\text { Excluídos por título } \\
\text { (5) }\end{array}$ & 0 & Scopus \\
\hline $\begin{array}{l}\text { (primary care) and } \\
\text { (single health service) } \\
\text { and (psychology) and } \\
\text { (mental health) }\end{array}$ & 5 & 5 & $\begin{array}{l}\text { Excluídos por título } \\
(5)\end{array}$ & 0 & Bireme \\
\hline $\begin{array}{l}\text { (primary care) and } \\
\text { (single health service) } \\
\text { and (psychology) and } \\
\text { (mental health) }\end{array}$ & 0 & - & - & - & Scielo \\
\hline Total & 175 & 173 & & 2 & Pubmed \\
\hline
\end{tabular}

Fonte: Desenvolvido pelos autores.

\section{Desenho dos estudos}

O primeiro estudo (Petterson et al.,2014) teve como objetivo verificar até que ponto as pessoas com problemas de saúde mental visitavam a atenção primária de saúde e subdividiram entre 4 padrões de cuidados, (a) apenas o setor de saúde mental, (b) apenas profissional da atenção primária, (c) cuidados duplos, profissional da atenção primária e setor de saúde mental e atenção primária, e (d) outras combinações de profissionais. A amostra foi composta por $(\mathrm{n}=184.636)$ participantes. Foi aplicado o questionário Mental Component score (MCS) of the Short Form - 12 (SF-12). O segundo estudo (Hoffman et al., 2019) apresentou como objetivos; (a) testar a viabilidade do uso de aplicativos de saúde mental para aumentar os serviços integrados de atenção primária; (b) solicitar feedback de pacientes e provedores para orientar a implementação, e (c) desenvolver um kit de ferramentas de aplicativos de saúde mental para disseminação em todo o sistema. Ao longo da duração de dois meses foram apresentados aplicativos de saúde mental para $(n=56)$ pacientes adultos. Cerca de cinquenta e quatro $(96,4 \%)$ pacientes concordaram em usar as ferramentas, enquanto $2(3,5 \%)$ pacientes recusaram (1 devido a questões de segurança e 1 devido a limitações de tempo). Os pacientes tinham de 18 a 70 anos, com média de 36,5 anos, e 37 (66,1\%) eram mulheres (Hoffman et al., 2019). 


\section{Aplicativos sobre Saúde Mental na Atenção Primária}

As ferramentas foram apresentadas a $35(62,5 \%)$ pacientes para ansiedade e problemas relacionados ao estresse e a 20 $(35,7 \%)$ pacientes para depressão. Oito $(14,2 \%)$ pacientes receberam aplicativos para tratar de problemas de sono e $3(5,3 \%)$ pacientes foram apresentados a aplicativos para outros problemas. Cerca de treze $(23,2 \%)$ dos 56 pacientes foram apresentados às ferramentas para várias condições (Hoffman et al., 2019). Dos 35 (64,8\%) pacientes que forneceram feedback sobre o uso das ferramentas, 7 (20\%) pacientes não conseguiram usar as ferramentas (3 devido a limitações do equipamento, 4 por outros motivos). Vinte e três $(65,7 \%)$ dos pacientes que forneceram feedback consideraram os aplicativos úteis. Vinte e quatro (68,5\%) dos pacientes relataram o uso de Breathe2Relax e 24 (68,5\%) relataram o uso de Auto ajuda para Gerenciamento de Ansiedade. Os pacientes usaram o Breathe2Relax para ajudá-los a dormir, para controlar a ansiedade e para se preparar para situações estressantes ou mentalmente desgastantes. Ao usar o Gerenciamento de Ansiedade, os pacientes tendem a acessar apenas alguns dos recursos que enfocam o alívio dos sintomas, educação sobre ansiedade, estratégias para controlar emoções fortes e rastreamento de humor (Hoffman et al., 2019).

\section{Atendimentos}

A maioria dos participantes $(52,6 \%)$ que obteve cuidados de saúde mental apenas do médico da atenção primária teve apenas uma consulta, em comparação com $16,9 \%$ das que obtiveram cuidados apenas no setor de saúde mental. Apenas $0,5 \%$ dos pacientes atendidos exclusivamente por médicos de atenção primária tiveram 21 ou mais consultas, em comparação com 13,2\% para aqueles atendidos exclusivamente no setor de saúde mental e 16,5\% para aqueles que obtiveram atendimento de ambos (Petterson et al.,2014).

Carca 8,1\% dos pacientes relataram pontuações MCS abaixo de 35, indicando saúde mental definida como ruim. Para este grupo, metade $(49,5 \%)$ obteve atendimento apenas com o médico da atenção primária, 5,0\% obteve atendimento apenas no setor de saúde mental e apenas 13,6\% receberam atendimento de ambos os profissionais. Entre os adultos que relataram ter uma consulta sobre saúde mental, 35,4\% ( $\mathrm{n}=3.979)$ tiveram consulta apenas com o médico da atenção primária, 43,7\% ( $\mathrm{n}=$ 4.938) obteve atendimento no setor de saúde mental, 8,5\% ( $\mathrm{n}=960)$ consultaram-se com ambos, e 12,5\% ( $\mathrm{n}=1411)$ consultaram-se com outro profissional (Petterson et al.,2014).

Após 1 ano da intervenção, que envolveu 32 profissionais do setor de saúde mental, 24 (75\%) completaram a pesquisa de acompanhamento. Dentro das funções integradas da equipe, nove (100\%) gerentes de cuidados, 12 (75\%) psicoterapeutas e $3(43 \%)$ consultores de psiquiatras responderam. O número de respostas para cada item da pesquisa variou. Vinte e três $(95,8 \%)$ profissionais responderam à pergunta da pesquisa sobre a utilização de ferramentas móveis com os pacientes; dezenove pessoas (82,6\%) indicaram que incorporam aplicativos em seu trabalho clínico. Cinco (25\%) membros da equipe de 20 indicaram que apresentam aplicativos para os pacientes 25-50\% do tempo, e 9 (45\%) introduzem ferramentas móveis $<25 \%$ do tempo. Ao usar aplicativos com pacientes, a condição abordada com mais frequência pela equipe foi ansiedade 20 (83,3\%), seguida de estresse 18 (75\%), depressão 14 (58,3\%), uso de álcool 6 (25\%) e uso de tabaco 6 (25\%) (Hoffman et al., 2019).

\section{Atenção Primária e Saúde Mental}

Os autores (Petterson et al.,2014) citam que problemas de saúde mental e condições específicas de saúde mental continuam prevalecendo na atenção primária. Um maior foco no cuidado centrado no paciente requer maior integração entre atenção primária e o setor de saúde mental para reduzir a fragmentação do cuidado e as disparidades nos resultados de saúde. Aplicativos de saúde mental são aplicáveis e relevantes para os pacientes em ambientes integrados de atenção primária em sistemas de saúde de rede de segurança. Os profissionais de saúde comportamental percebem o valor clínico do uso dessas 
ferramentas como parte do atendimento ao paciente, mas exigem treinamento para aumentar seu nível de conforto e confiança ao aplicar essas ferramentas aos pacientes. Para aumentar o envolvimento do provedor e do paciente, os aplicativos móveis devem ser acessíveis, simples, intuitivos e diretamente relevantes para as necessidades de tratamento dos pacientes (Hoffman et al., 2019).

As características principais das pesquisas selecionadas para este estudo, encontram-se expostas na Tabela 4.

Tabela 4. Síntese dos artigos incluídos.

\begin{tabular}{|c|c|c|c|c|c|}
\hline $\begin{array}{c}\text { Autor/ Ano/ } \\
\text { Local de } \\
\text { publicação }\end{array}$ & Objetivo & $\mathbf{n}$ & Método & Resultados & Conclusão \\
\hline $\begin{array}{c}\text { Petterson et } \\
\text { al., } 2014 \\
\text { Estados } \\
\text { Unidos }\end{array}$ & $\begin{array}{c}\text { Verificar até } \\
\text { que ponto as } \\
\text { pessoas com } \\
\text { problemas de } \\
\text { saúde mental } \\
\text { visitavam a } \\
\text { atenção } \\
\text { primária de } \\
\text { saúde }\end{array}$ & 184.636 & $\begin{array}{l}\text { Questionário } \\
\text { Mental } \\
\text { Component } \\
\text { score (MCS) of } \\
\text { the Short Form } \\
\text { - } 12 \text { (SF-12) }\end{array}$ & $\begin{array}{l}\text { A maioria dos participantes }(52,6 \%) \\
\text { que obteve cuidados de saúde mental apenas do } \\
\text { médico da atenção primária teve apenas uma } \\
\text { consulta, em comparação com } 16,9 \% \text { das que } \\
\text { obtiveram cuidados apenas no setor de saúde } \\
\text { mental. Apenas } 0,5 \% \text { dos pacientes atendidos } \\
\text { exclusivamente por médicos de atenção } \\
\text { primária tiveram } 21 \text { ou mais consultas, em } \\
\text { comparação com } 13,2 \% \text { para aqueles atendidos } \\
\text { exclusivamente no setor de saúde mental e } \\
16,5 \% \text { para aqueles que obtiveram atendimento } \\
\text { de ambos }\end{array}$ & $\begin{array}{l}\text { Problemas de saúde mental e } \\
\text { condições específicas de } \\
\text { saúde mental continuam } \\
\text { prevalecendo na atenção } \\
\text { primária. Um maior foco no } \\
\text { cuidado centrado no paciente } \\
\text { requer maior integração entre } \\
\text { atenção primária e o setor de } \\
\text { saúde mental para reduzir a } \\
\text { fragmentação do cuidado e as } \\
\text { disparidades nos resultados de } \\
\text { saúde. }\end{array}$ \\
\hline $\begin{array}{l}\text { Hoffman et } \\
\text { al., } 2019 \\
\text { Austrália }\end{array}$ & $\begin{array}{c}\text { (a) } \\
\text { testar a } \\
\text { viabilidade do } \\
\text { uso de } \\
\text { aplicativos de } \\
\text { saúde mental } \\
\text { para aumentar } \\
\text { os serviços } \\
\text { integrados de } \\
\text { atenção } \\
\text { primária; (b) } \\
\text { solicitar } \\
\text { feedback de } \\
\text { pacientes e } \\
\text { provedores para } \\
\text { orientar a } \\
\text { implementação, } \\
\text { e (c) } \\
\text { desenvolver um } \\
\text { kit de } \\
\text { ferramentas de } \\
\text { aplicativos de } \\
\text { saúde mental } \\
\text { para } \\
\text { disseminação } \\
\text { em todo o } \\
\text { sistema. }\end{array}$ & 88 & $\begin{array}{c}\text { Uso e } \\
\text { aplicativos } \\
\text { (Auto ajuda e } \\
\text { Breathe2Relax) }\end{array}$ & $\begin{array}{c}\text { Dos } 35(64,8 \%) \text { pacientes que forneceram } \\
\text { feedback sobre o uso das ferramentas, } 7(20 \%) \\
\text { pacientes não conseguiram usar as ferramentas } \\
\text { ( } 3 \text { devido a limitações do equipamento, } 4 \text { por } \\
\text { outros motivos). Vinte e três }(65,7 \%) \text { dos } \\
\text { pacientes que forneceram feedback } \\
\text { consideraram os aplicativos úteis. Vinte e } \\
\text { quatro }(68,5 \%) \text { dos pacientes relataram o uso } \\
\text { de Breathe2Relax e } 24 \text { (68,5\%) relataram o uso } \\
\text { de Auto ajuda para Gerenciamento de } \\
\text { Ansiedade. Dentro das funções integradas da } \\
\text { equipe, nove (100\%) gerentes de cuidados, } 12 \\
\text { (75\%) psicoterapeutas e } 3 \text { (43\%) consultores de } \\
\text { psiquiatras responderam. Vinte e três }(95,8 \%) \\
\text { profissionais responderam à pergunta da } \\
\text { pesquisa sobre a utilização de ferramentas } \\
\text { móveis com os pacientes; dezenove pessoas } \\
\text { ( } 82,6 \%) \text { indicaram que incorporam aplicativos } \\
\text { em seu trabalho clínico. Cinco ( } 25 \%) \text { membros } \\
\text { da equipe de } 20 \text { indicaram que apresentam } \\
\text { aplicativos para os pacientes } 25-50 \% \text { do } \\
\text { tempo, e } 9 \text { (45\%) introduzem ferramentas } \\
\text { móveis <25\% do tempo. Ao usar aplicativos } \\
\text { com pacientes, a condição abordada com mais } \\
\text { frequência pela equipe foi ansiedade } 20 \\
\text { ( } 83,3 \%) \text {, seguida de estresse } 18 \text { (75\%), } \\
\text { depressão } 14 \text { (58,3\%), uso de álcool } 6 \text { ( } 25 \%) \text { e } \\
\text { uso de tabaco } 6 \text { ( } 25 \%)\end{array}$ & $\begin{array}{l}\text { Aplicativos de saúde } \\
\text { mental são aplicáveis e } \\
\text { relevantes para os pacientes } \\
\text { em ambientes integrados de } \\
\text { atenção primária em sistemas } \\
\text { de saúde de rede de } \\
\text { segurança. Os profissionais de } \\
\text { saúde comportamental } \\
\text { percebem o valor clínico do } \\
\text { uso dessas ferramentas como } \\
\text { parte do atendimento ao } \\
\text { paciente, mas exigem } \\
\text { treinamento para aumentar } \\
\text { seu nível de conforto e } \\
\text { confiança ao aplicar essas } \\
\text { ferramentas aos pacientes. } \\
\text { Para aumentar o } \\
\text { envolvimento do provedor e } \\
\text { do paciente, os aplicativos } \\
\text { móveis devem ser acessíveis, } \\
\text { simples, intuitivos e } \\
\text { diretamente relevantes para as } \\
\text { necessidades de tratamento } \\
\text { dos pacientes }\end{array}$ \\
\hline
\end{tabular}

Fonte: Petterson et al., 2014; Hoffman et al. (2019).

$\mathrm{Na}$ análise e discussão dos artigos, foi possível verificar que tanto para Petterson et al. (2014) quanto para Hoffman et al. (2019), objetivar uma assistência mais humana na AB segue sendo um tema a ser discutido quando trata-se de SM, onde a não fragmentação dos atendimentos quando um paciente chega em busca de uma solução para os seus incômodos psicológicos 
e físicos, é algo a ser objetivado e a APS pode ir em busca de um atendimento mais integrado quando trata-se de SM e atendimento ao paciente. Os autores concordam que por mais que tenha evoluído a maneira de atender os pacientes, o número de profissionais qualificados e conhecimento destes, precisa ser ampliado para que haja mais integração entre os cuidados do tratamento da SM e o acolhimento feito nas APS, bem como torna-se necessário ter a consciência de que nem todos os pacientes possuem habilidades necessárias para lidar com as inovações tecnológicas do momento, embora isto seja um "gol" atingido pela grande maioria dos que buscam a $\mathrm{AB}$.

A utilização de Aplicativos de SM vem a somar no mercado do campo da saúde, sendo esta uma ferramenta utilizada por vários profissionais como forma de obter melhores resultados no campo psicológico, mesmo que alguns pacientes encontrem barreiras para a utilização destas ferramentas, este tem sido um meio para poder tornar a integração do trabalho nas APS com a SM, algo mais possível e produtivo na aquisição dos resultados no campo psicológico, além do aumento da adesão de pessoas à este tipo de tratamento (Hoffman et al., 2019).

Para a OMS (Organização Mundial da Saúde) a saúde mental não é apenas um fato isolado mas é um fato que engloba o lado psicológico, psiquiátricos e fatores sociais que irão compor um trabalho multidisciplinar quando falamos de saúde mental, enquanto que a psiquiatria por muito tempo ligava a saúde mental à transtornos psiquiátricos tratando este tema como algo ligado diretamente aos modelos medicina, e assim a saúde mental foi vista por muito tempo, o que aos poucos esta visão foi mudando para uma forma onde o paciente deixa de ser somente um ser com cérebro mas passa a ser uma pessoa que tem aspectos físicos, psicológicos e sociais que irão influenciar no estilo de vida que leva e consequentemente que afetam o seu estado de saúde mental (Carrazana, 2003).

Nos estudos de Hoffman et al. (2019) não foi possível controlar muitas das variáveis que desejavam os pesquisadores fazer, e dentro de uma delas foi obter resultados sobre o nível de gravidade que cada paciente se encontrava ou nível de envolvimento de cada médico no período de observação ao estado de cada paciente, dentre outros temas observados nos resultados, o que não permitiu que a pesquisa tivesse um resultado preciso já que o estudo foi feito em um estilo padrão para um determinado tipo de comunidade implicando desta forma, em uma certa dificuldade em quantificar o número de médicos e pacientes que realmente desejam utilizar algum tipo de ferramenta móvel no tratamento do atendimento primário de saúde, até mesmo porque não foi detectado um estilo padrão unificado onde todo o sistema de saúde pudesse utilizar além das diferentes atualizações no sistema ademais, estas atualizações nas ferramentas móveis dava-se de forma singular para cada região onde se utilizava o atendimento básico segundo a necessidade prestada à população na área da saúde mental, porém Kates et al. (2018) vê as diferentes possibilidades de comunicação com o paciente por vias tecnológicas um avanço na atenção primária que atinge comunidades que não tem como adquirir acesso à especialistas da área da saúde mental e mesmo na singularidade de cada região, um número maior de pacientes pode ser atendida em um espaço de tempo melhorado, saindo da limitação do número de pessoas que podem ser atendidas em uma clínica versus a positividade do número de pessoas que podem ser atingidas fora do espaço clinico, sendo desta forma o atendimento básico feito por profissionais qualificados e de maneira integrada, algo com potencial para atender um público muito maior e com mais eficiência do que os padrões antigos utilizados nos espaços clínicos.

Em contra ponto pode-se observar com este estudo que um dispêndio de trabalho dos profissionais da área da saúde na $\mathrm{AB}$ será maior na integração proposta ou pelo menos é assim que consideram alguns trabalhadores da $\mathrm{ABP}$, já que por não entenderem o processo completo deste tipo de integração, uma vez que não participaram da construção deste trabalho na área da SM e enfrentam muitas vezes a barreira da falta de instrumentalização nos locais de trabalho, sentem-se preocupados com a possibilidade de enfrentar situações extremas como problemas psicopatológicos ou atender pacientes que tenham tido experiências com tentativas de suicídio o que por sua parte, exigiria além de um melhor preparo para atender estes casos, um 
aumento na sua carga horária de trabalho o que os faz dar preferência ao assistencialismo ao em vez de superá-lo diminuindo a barreira da medicalização nos consultórios (Gryschek \& Pinto, 2015).

Nos Estados Unidos da América foi utilizada o modo de atendimento 5A'S (Five Psychological Domains), que consiste em atender ao paciente de uma maneira mais ampla onde ele não somente será avaliado pelos sintomas apresentados, mas passará por um processo integrado de atendimento variando conforme o problema de cada paciente que procura a $\mathrm{AB}$, havendo um trabalho que envolve uma perspectiva psicológica, social e biológica que analisa como um todo o que pode estar provocando o sintoma do paciente, levando o profissional da ABP a dar informações sobre as opções de mudanças que cada paciente pode fazer na sua vida para obter melhor resultados no campo da SM além disso, a coleta de dados vai desde fatores psicológicos até a obtenção de informações sobre o a interação destes pacientes no ambiente que vive com seus familiares e pessoas que convive ao seu redor sendo esta uma maneira que não impõe uma solução imediata, mas oferece opções onde o próprio paciente pode discutir as diferentes maneiras e ações a tomar para resolver o problema proposto em questão e que o fez procurar ajuda, mas em caso que não goste de nenhuma das opções ofertadas pelo profissional que o atendeu na AB, o próprio paciente pode fazer as suas sugestões para poder encontrar soluções ao seu problema e será escutado ao fazer estas sugestões acontecendo desta forma, uma maior integração entre paciente e profissional da saúde na ABP que fará este atendimento de forma mais personalizada (Hunter, 2016).

Além dos esforços dos profissionais e todas as pessoas envolvidas na integração, para que houvesse melhores possibilidades de desenvolvimento de trabalhos essenciais para que este sistema funcionasse, para o Chile foi muito importante o apoio governamental, uma vez que políticas de planos de apoio à nível nacional foram necessárias para que tal sistema de saúde fosse prioridade em cada APS onde foi aplicada essa integração. Mesmo tendo criado um programa nacional de saúde mental em 1966 ainda que não implementado no Chile, já era alvo de discussão a importância de criar-se uma integração da saúde mental em cada APS porém, poucos anos depois houveram as consequências de retalhos neste programa devida a ditadura militar imposta em 1973, o que tornou o processo de progresso mais lento para então somente na década de 1990 serem retomados as análises feitas anteriormente contando com o apoio político necessário para este desenvolvimento, o que possibilitou o tracejo de metas sendo uma delas que em 2020 houvesse uma diminuição da quantidade de pessoas com incapacidades mentais na sociedade, através da integração da saúde mental em cada APS com o apoio governamental e o objetivo de que os pacientes pudessem ter a oportunidade resolver o seu problema de forma mais rápida e eficaz no que tange à saúde mental deixando claro para este País, a importância do envolvimento de políticas públicas para o bom desenvolvimento da saúde mental no programa de integração em APS (Minoletti, Rojas \& Hovitz-lennon, 2012).

Atendimentos a casos com depressão tendem a ter uma similitude quando o paciente chega à APS para ser atendido, o que de certa forma propicia uma facilidade no diagnóstico deste tipo de enfermidade porém cada clínica, segundo o estudo feito por Fickel, Yano e Parker (2009) tem a sua própria individualidade, tanto na maneira em como receber os pacientes bem como na maneira de tratá-los, embora cada local necessite dos seus próprios cuidados já que tem as suas características próprias e necessidades locais, mas o que chama a atenção e contradiz o que os demais estudos mostram, é que estes autores afirmam que em alguns casos ainda o primeiro passo a ser dado em diagnósticos de depressão é a receita de anti-depressivos, pouca atenção ao paciente e pouco acompanhamento posterior ao caso devido a grande demanda de pessoas e muitas vezes a pouca informação adequada sobre depressão, o que pode acarretar em um diagnóstico precipitado sem levar em conta que cada pessoa tem diferentes níveis de depressão, sendo muitas vezes diagnósticos feitos de maneira superficial onde possíveis intenções de suicídios e outros casos graves muitas vezes não são detectados na triagem, incorrendo assim em um problema para que a integração seja bem sucedida na sua totalidade, o que para isto acontecer seria necessário um maior monitoramento dos profissionais que fazem o atendimento na $\mathrm{AB}$ e melhores diretrizes de atendimento. 
A forma de aprender para poder atender na ABP mudou e o que antes era tido como primordial em forma de conceitos no aprendizado, agora torna-se conhecimento prático, imprescindível para este tipo de atendimento onde os problemas vem antes de qualquer estabelecimento de conceito, já que esses problemas irão dizer se o que foi aprendido é algo a ser confirmado ou se há contradição surgindo assim, novas maneiras de tratar os pacientes e abrindo campo para novas descobertas (Borochovicius \& Tortella, 2014).

Em resumo, a eficácia de um atendimento de qualidade na atenção primária de saúde que tenha recursos apropriados para este atendimento, com um nível de aceitação pública que venha a utilizar estes recursos, é um progresso na área da saúde mental e um avanço no atendimento à um número maior de pacientes, principalmente em áreas onde os recursos econômicos para atendimentos com profissionais especializados na saúde mental é algo mais difícil de ser atingido, desta forma um grupo maior de pacientes podem ser beneficiados (Kates et al., 2018).

\section{Considerações Finais}

A maioria dos participantes que obtiveram cuidados de saúde mental apenas do médico da atenção primária, tiveram somente uma consulta demonstrando desta forma, a necessidade de uma maior integração entre a atenção primária e o setor de saúde mental buscando-se assim, haver uma redução na fragmentação do cuidado com o paciente bem como os resultados obtidos nestas consultas.

Ao tratar-se de atualização no uso de ferramentas eletrônicas, tanto por parte de profissionais da saúde como por parte dos pacientes como forma de integrar estes sistemas para haver melhores resultados para o paciente, os estudos demonstraram boa aceitação neste tipo de método, embora ainda haja algumas barreiras para alguns pacientes, o que incita a busca por aplicativos de saúde mental de menor complexidade para o envolvimento de uma possível totalidade da população que busca um atendimento na área da saúde mental. A partir disso, são necessários a realização de trabalhos futuros para abordarem melhor o assunto que ainda permanece pouco discutido na literatura.

\section{Referências}

Carrazana, V.(2003). El concepto de salud mental en psicologia humanista-existencial. Ajayu Órgano de Difusión Científica del Departamento de Psicología UCBSP.

Carvalho, J.L.S. \& Maria P.S.S.N. (2017). Práticas integrativas e complementares como recurso de saúde mental na Atenção Básica.Revista Gaúcha de Enfermagem, 38(4).

Fickel, J. J. \& Yano. (2009). Clinic-level process of care for depression in primary care settings. Administration and Policy in Mental Health, (36).

Gryschek, G. \& Pinto, A. A. M.(2015). Saúde Mental: como as equipes de Saúde da Família podem integrar esse cuidado na Atenção Básica?. Revista Ciência e Saúde Coletiva, (20).

Hoffman, L., Benedetto, E., Huang, H., Grossman, E., Kaluma, D., Mann, Z., \& Torous, J. (2019). Augmenting mental health in primary care: A 1-year study of deploying smartphone apps in a multi-site primary care/behavioral health integration program. Frontiers in psychiatry, 10(94).

Hunter, L. C., Goodie, J. L., Oordt, M. S. \& Dobmeyer, A. C. (2016). Anne C. Integrated Behavioral Healt in Primary Care: Step-by-step guidance for assessment and intervention. American Psychological Association. (2a ed.).

Minolletti, A., Rojas, G . \& Horvitz-Lennon, M. (2012). Salud mental en atención primária en Chile: aprendizajes para Latinoamérica. Revista Ciências \& Saúde Coletiva.

Moher D, Shamseer L \& Clarke M. (2015). Preferred reporting items for systematic review and meta-analysis protocols (PRISMA-P) 2015 statement. Systematic Review, 4(1), 1.

Pithon M. M., Sant'anna L. I. D. A., Baião F. C. S., Santos R. L., Coqueiro R. S. \& Maia L C. (2015). Assessment of the effectiveness of mouthwashes in reducing cariogenic biofilm in orthodontic patients: a systematic review, 43,297-308.

Petterson, S., Miller, B. F., Payne-Murphy, J. C., \& Phillips Jr, R. L. (2014). Mental health treatment in the primary care setting: Patterns and pathways. Families, Systems, \& Health, 32(2), 157. 
Research, Society and Development, v. 10, n. 3, e43210313394, 2021

(CC BY 4.0) | ISSN 2525-3409 | DOI: http://dx.doi.org/10.33448/rsd-v10i3.13394

Silva Filho, J. A., \& de Moraes Bezerra, A. (2018). Acolhimento em Saúde Mental na Atenção Primária à Saúde: Revisão Integrativa. Revista de Psicologia, $12(40), 613-627$.

Wenceslau, L.D. \& Francisco O. (2015). "Saúde mental na atenção primária e Saúde Mental Global: perspectivas internacionais e cenário brasileiro." Interface-Comunicação, Saúde, Educação, 1121-1132. 